\begin{tabular}{|l|l|l|l|l|l|}
\hline MUNIBE Antropologia-Arkeologia & $n^{\circ} 69$ & $297-309$ & DONOSTIA & 2018 & ISSN 1132-2217 • eISSN 2172-4555 \\
\hline
\end{tabular}

\title{
Estudio antropológico de un individuo hallado en el edificio religioso medieval de la plaza Eduardo de Castro (Astorga, España). ¿Muerte accidental o intencionada?
}

\author{
Anthropological study on an individual found out in the mediaeval \\ religious building of the Eduardo de Castro Square (Astorga, Spain). \\ Accidental or intentional death?
}

PALABRAS CLAVES: Estratigrafía, fractura, perimortem, postmortem, carbonización

GAKO-HITZAK: Estratigrafia, haustura, perimortem, postmortem, karbonizazioa.

KEY WORDS: Stratigraphy, fracture, perimortem, postmortem, carbonisation.

\section{Susana GÓMEZ-GONZÁLEZ(1) y Alejandro MARTíN-LÓPEZ ${ }^{(2)}$}

\section{RESUMEN}

Diferenciar entre fracturas perimortem y postmortem no es tarea fácil de desempeñar, por lo que las características macroscópicas que pueden observarse en una fractura perimortem son claves a la hora de diferenciarla de una postmortem. Sin embargo, establecer una relación casuística entre el momento de la muerte y la lesión resulta complicado, ya que el contenido de humedad del hueso se mantiene tiempo después de la muerte, mostrando, por lo tanto, propiedades similares al tejido esquelético fresco, por lo que es necesario atender a otras evidencias, intrínsecas y extrínsecas, que nos permitan aproximarnos a la causa del suceso. En este trabajo, mediante la aproximación a la causa de muerte del Individuo 6, trata de evidenciar la importancia de la interacción entre la antropología física y la arqueología, con el fin de inferir en la interpretación de un suceso de estas características.

\section{LABURPENA}

Perimortem eta postmortem hausturak bereiztea ez da lan erraza; hori dela eta, perimortem haustura batean ikus daitezkeen ezaugarri makroskopikoak funtsezkoak izaten dira postmortem haustura batetik bereizteko garaian. Dena den, zaila izaten da heriotzaren unearen eta lesioaren arteko erlazio kasuistikoa zehaztea; izan ere, hezurraren hezetasunaren edukia hil ondoren luzaroan mantentzen da eta, horrenbestez, eskeleto-ehun freskoaren antzeko ezaugarriak mantentzen ditu eta beharrezkoa izaten da gertakariaren kausara gerturatzea ahalbidetuko liguketen beste ebidentzia batzuetan (intrintsekoak eta estrintsekoak) arreta jartzea. 6. banakoaren heriotza-kausara egindako gerturatze-lanaren bidez, antropologia fisikoaren eta arkeologiaren arteko elkarrekintzak duen garrantzia erakutsi nahi dugu ezaugarri horiek dituen gertakizun baten interpretazioan eragin ahal izateko.

\section{ABSTRACT}

Astorga (León, Spain) is one of the most ancient Christian bishopric in the Iberian Peninsula. There are not many remains form the Early Christian period in this geographical area. Nevertheless, the discovery of a church during the archaeological works around the Gothic Cathedral constitutes the oldest testimony of Christian building in the city. During these archaeological works, a human male body was found on the floor of the church. It was named Individual number 6 , and his death is the principal aim of this paper.

Distinguishing between perimorten and postmorten fractures is not an easy task due to the key macroscopic characteristics which can be appreciated in perimortem fractures to differentiate from the postmortem ones. However, a link is made between the time of dead and the lesion which is extremely difficult. The dampness of the bone has lasted after the time of death, thus showing similar properties such as fresh skeletal tissue. For this reason, it is necessary take care of intrinsic and extrinsic evidence in order to analyse the cause of death.

In this work-study, an approximation of the cause of death of number 6, stresses the importance of the interaction between physical anthropology and archaeology with the aim of interpreting the events of these characteristics.

\section{INTRODUCCIÓN}

La vinculación existente entre la arqueología y la antropología, confiere, científicamente hablando, la posibilidad de interpretar sucesos acaecidos en la antigüedad, que de manera individual resultaría complejo e incluso imposible. El trabajo que presentamos es un claro ejemplo de ello, permitiéndonos comprender con más certeza una parte de la historia de la ciudad de Astorga.

\footnotetext{
(1) Área de Antropología Física. Facultad de CC. Biológicas y Ambientales. Universidad de León. Susana Gómez González. Área de Antropología Física. Facultad de CC. Biológicas y Ambientales. Universidad de León. Campus de Vegazana s/n. 24007-León, España. sgomg@unileon.es (2) Arqueólogo.
} 
Un claro ejemplo de esta relación se refleja en el primer anexo de la publicación de Carter (1987), donde Douglas E. Berry presenta un primer informe forense sobre el cuerpo del joven faraón que su compatriota había extraído de los sucesivos sarcófagos de la KV 62. Este escocés, con formación en medicina, dedicó gran parte de su vida científica a la aplicación de la antropología forense al estudio de restos humanos procedentes de yacimientos arqueológicos. Tal vez resulte anecdótico, pero a la sombra de los grandes descubrimientos egipcios de las primeras décadas del siglo XX resultó evidente que la colaboración entre arqueólogos y antropólogos forense era absolutamente necesaria. Asimismo, en los últimos cuarenta años, los adelantos acontecidos en diferentes campos como la paleopatología, la paleodieta y la genética, han facilitado, tanto a la arqueología como a la antropología la posibilidad de caracterizar con mayor exactitud las poblaciones humanas del pasado, su alimentación y sus enfermedades, así como inferir en las diferentes causas de muerte. Siguiendo el espíritu de colaboración científica cultivada durante estas décadas, se abordó el estudio complementario desde ambos puntos de vista de los restos humanos pertenecientes al Individuo 6 (I.6), localizado en el yacimiento de la Plaza Eduardo de Castro (PEC), en la capital maragata.

En la reducida área del yacimiento PEC se localizaron una cantidad importante de restos humanos. La mayoría de los cuerpos tienen una relación directa con el uso funerario que durante siglos tuvo el solar, como ha quedado demostrado no solo en esta intervención, sino también en seguimientos puntuales dentro del atrio de la Catedral (Muñoz, 2001), que se encuentra en las inmediaciones. Sin embargo, el 1.6 es localizado sobre el pavimento de la iglesia alto medieval que, antes que las sucesivas necrópolis, se construyó en esta área de la ciudad. El trabajo realizado, combinando el análisis estratigráfico y el estudio antropológico, tratará de resolver la causa de muerte de este individuo, y dilucidar si esta fue accidental o intencionada.

\section{CONSIDERACIONES PREVIAS}

\subsection{Contexto histórico}

La llegada del cristianismo a la Península Ibérica es uno de los campos de investigación sobre el que los historiadores de la antigüedad han profundizado más en los últimos treinta años. Independientemente de las diferentes hipótesis propuestas (Blázquez, 1986; DíazMartínez et al., 2007; Teja, 1990), es evidente que el papel que las diócesis de Mérida y Astorga tienen en este proceso es capital. A mediados del siglo III d. C., y en respuesta a una carta que desde ambas diócesis mandan sus fieles, Cipriano de Cartago contesta proponiendo una serie de cambios en ambas sedes en su Carta LXVII (Gil-Tamayo, 2013). Este documento, es considerado el más temprano de la historia de la Hispania cristiana y presenta comunidades complejas entorno a ambas ciudades.
Astorga ocupa por lo tanto un papel principal en la historia de la expansión del cristianismo en el Noroeste peninsular, gobernando una diócesis que ocupa desde los cauces del Duero y Esla hasta el Bierzo actual. Esta Diócesis será una de las estructuras administrativas más estables, hasta que bien entrada la Edad Media, surjan diócesis nuevas como Oviedo, León o Zamora. Durante este periodo Astorga transformó su capitalidad del convento jurídico, en la sede de una nueva forma de religiosidad y de administración: una sede episcopal. Es en este contexto histórico de constantes transformaciones edilicias, en el que se construye la iglesia en cuyo pavimento fue localiza I.6.

\subsection{Contexto arqueológico}

Sin embargo, la abundante bibliografía histórica sobr la Diócesis, no tiene paralelo en la bibliografía arqueológica. Solamente dos yacimientos arqueológicos han sido confirmados como espacios religiosos cristianos: Marialba de la Ribera (cc. Siglos IV-VII d.C) (Hauschild, 1968,1970) y el que es objeto de este estudio, situado en la PEC de Astorga. Probablemente, junto con estos dos yacimientos habría que incluir una parte de la villa rural de Navatejera (Benéitez y Miguel, 1993) pero sin embargo la funcionalidad religiosa es tan solo una hipótesis. El resto de elementos de la cultura material relacionados con el cristianismo desde el siglo III al IX d. C., han sido depositados en diversos museos (Museo Catedralicio de Astorga, Museo de los Caminos de Astorga, Museo Provincial de León, Museo Provincial de Zamora), privados de un contexto arqueológico que nos permita entender más características de la Diócesis en sus primeros siglos de existencia, hasta su refundación.

Este edificio religioso de la PEC, se encuentra en el ángulo Noreste del recinto amurallado del solar romano, en las inmediaciones de una de las puertas originales que conserva la muralla. Actualmente, este espacio constituye el área que separa la Catedral de Astorga, el palacio episcopal y la cabecera de la iglesia de Santa Marta. Las excavaciones arqueológicas en PEC fueron desarrolladas como parte de un plan de reurbanización del casco histórico de la ciudad. Bajo la dirección de la arqueóloga municipal $M^{a}$. A. Sevillano, se llevó a cabo la intervención técnica entre noviembre y diciembre de 1999 (Sevillano y Vidal, 2001). Una vez terminados los trabajos arqueológicos y protegidas las estructuras principales conservadas in situ, el yacimiento quedó cubierto por el acceso Sureste al atrio de la catedral (Fig. 1).

Se documentó una secuencia de más de ochenta Unidades Estratigráficas (UE). A partir del año 2015 comienza una revisión de la documentación y material arqueológico, así como de los restos humanos conservados en el Museo Romano de Astorga, cuyo primer estadio consistió en organizar las UE agrupándolas en nueve fases, correspondientes a las diferentes funciones que ha tenido el solar. 


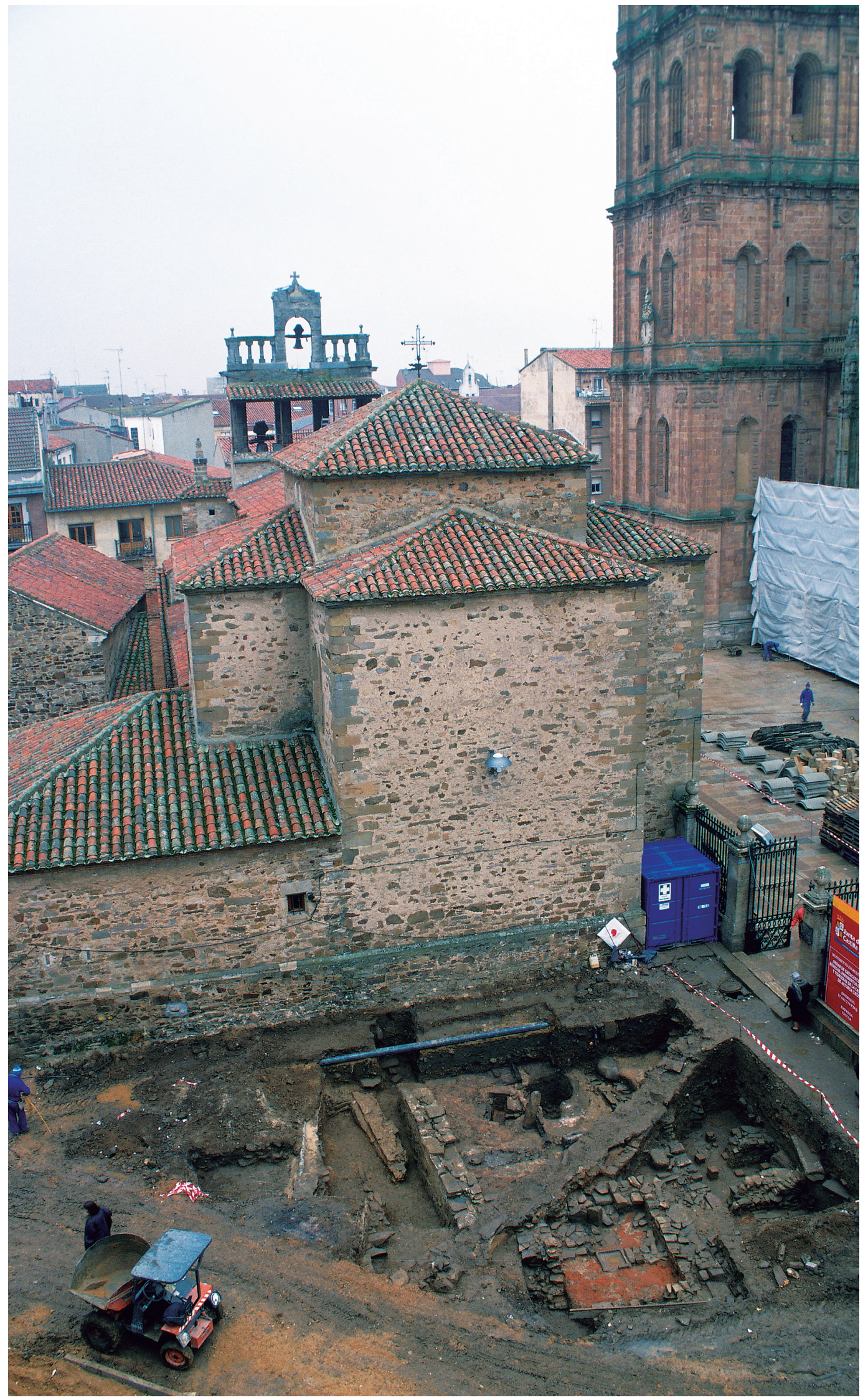

Fig. 1. Fotografía de las excavaciones arqueológicas de la plaza Eduardo de Castro, con la iglesia de Santa Marta y la Catedral de Astorga al fondo. Fotografia: $\mathrm{M}^{\mathrm{a}}$. Ángeles Sevillano. I Photography of the archaeological works in the Eduardo de Castro square. In the bottom Santa Marta's church and the Cathedral of Astorga. Photography: $\mathrm{M}^{\mathrm{a}}$. Ángeles Sevillano. 
Una vez individualizadas cada una de las fases y su relación estratigráfica el trabajo de investigación se centra en la Fase III y IV, profundizando en la funcionalidad que tuvo el edificio religioso dentro de la organización de la primitiva diócesis de Astorga, así como en las razones y proceso de desaparición de la iglesia. Es precisamente en las UEs incluidas en la Fase IV donde aparece el I.6 (Tabla I).

\begin{tabular}{|l|c|}
\hline FASES & FUNCIONES \\
\hline Fase IX & Saneamiento contemporáneo \\
\hline Fase VIII & Estructuras hortícolas siglo XVIII y XIX \\
\hline Fase VII & Osario bajomedieval \\
\hline Fase VI & Necrópolis II \\
\hline Fase V & Necrópolis I \\
\hline Fase IV & Derrumbe y abandono de un edificio religioso \\
\hline Fase III & Edificio religioso cristiano (ss. VII-IX) \\
\hline Fase II & Amortización de edificio termal altoimperial \\
\hline Fase I & Edificio termal altoimperial \\
\hline
\end{tabular}

Tabla 1: Relación de fases de acuerdo al uso de ocupación del yacimiento / Stages list and theirs funcionalities.

\section{CONTEXTO ARQUEOLÓGICO DE I.6}

Durante la excavación arqueológica de PEC en 1999, se localizaron multitud de restos humanos relacionados con el uso funerario que en dos fases (Fase V y VI) tuvo este solar. La necesidad de sistematizar el estudio y la extracción de los restos humanos dentro de una intervención urbanística generó una nomenclatura que incluía un número identificativo del individuo, acompañado del número de enterramiento al que estaba asociado (varios individuos pueden estar rela-

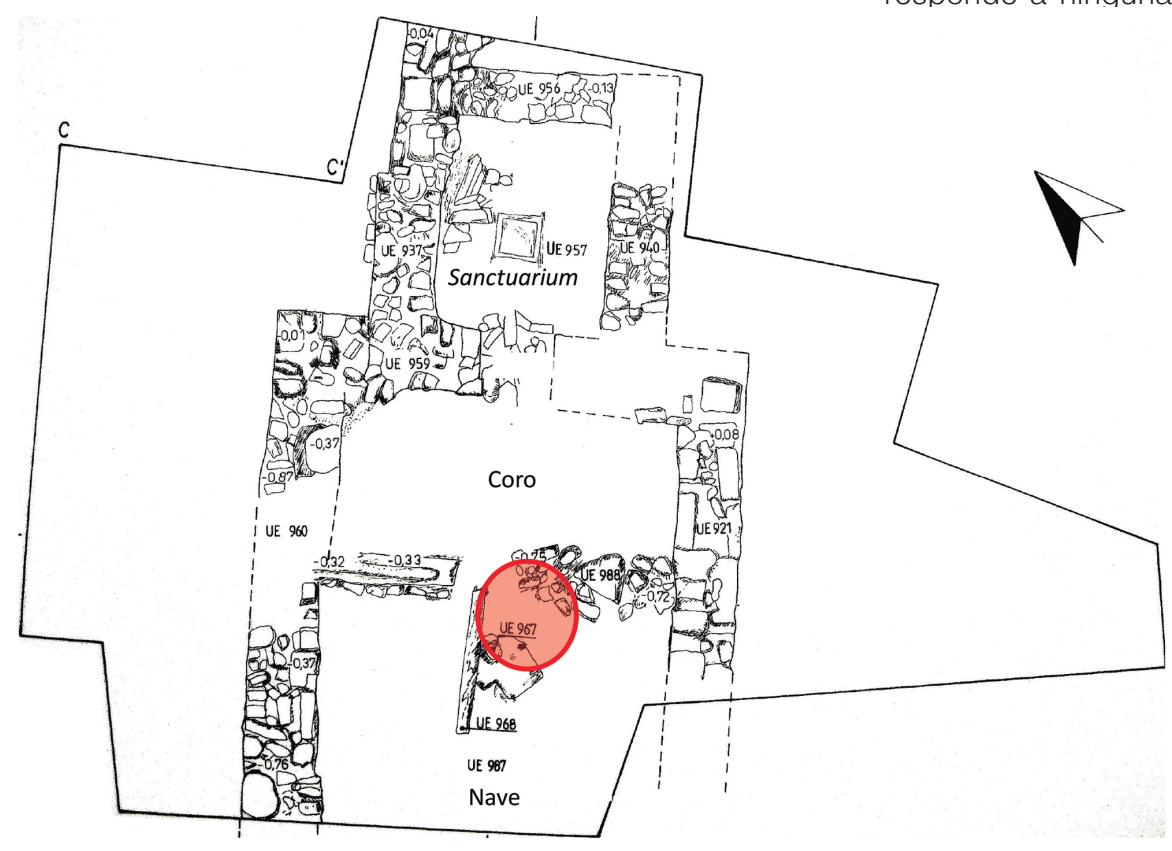

cionados con el mismo enterramiento). Sin embargo y aunque no existe una conexión estratigráfica directa entre las dos necrópolis y el I.6, este fue identificado originalmente como I.6/T.9.

El I.6 fue localizado en la frontera estratigráfica entre la Fase III (uso litúrgico de un espacio religioso) y la Fase IV (abandono y destrucción de la misma). Situado en el interior del edificio religioso, el cuerpo descansa decúbito supino en el área de transición entre la parte de la nave destinada a los fieles y el coro, desde donde los miembros del clero que no ofician la eucaristía asistían a los oficios religiosos.

Estratigráficamente, el cuerpo se apoya directamente sobre el pavimento de la nave (UE 987- Fase III). Sobre el extremo distal de la mano derecha se localizan los restos de una viga calcinada (UE 968 Fase IV), que debía formar parte de la estructura de cubierta del edificio. Tanto I.6, como los restos de viga, así como el resto del pavimento del interior de la nave, están cubiertos por una UE de gran potencia (UE 923 - Fase IV), donde la presencia de material constructivo removido de su posición original, entre ellos abundantes fragmentos de teja y una importante presencia de carbones, e incluso el color llamativamente oscuro de la tierra, nos ha llevado a identificarla como correspondiente al momento principal del colapso de los muros y la cubierta del edificio, relacionándolo con un posible incendio que puntualmente se produjo en el interior de la nave. Sin embargo, no aparecen evidencias de este incendio ni en las UE de la habitación anexa sanctuarium, ni en las referidas al exterior del edificio.

El análisis de la posición estratigráfica del Individuo 6 parece indicar que, a falta del estudio antropológico, el cuerpo se depositó en el área de los canceles que separan la nave del coro de forma accidental (no responde a ninguna forma de inhumación) en un momento en el que el pavimento de la iglesia estaba aun a la vista. En un momento inmediatamente posterior parte de la cubierta cae sobre el pavimento y el I.6. Posteriormente el resto de material decorativo, sillarejo de los muros y restos de cubierta, así como los restos de material calcinado cubren tanto el cuerpo, como el pavimento del templo (Fig. 2)

Fig. 2. Plano de la iglesia con la indicación de las áreas litúrgicas. En rojo el área donde el Individuo 6 fue localizado. Plano: $\mathrm{M}^{\mathrm{a}}$ Ángeles Sevillano. Edición: Autores. / Plan of the church with the liturgical areas mark. In red the area where the Individual 6 was found out. Plan: Mª́ngeles Sevillano. Edition: Authors. 


\section{LESIONES EN EL INDIVIDUO 6 (I.6)}

\subsection{Patrones de fractura perimortem vs postmortem}

A la hora de interpretar la causa de muerte de un individuo, son diferentes los parámetros a tener en cuenta, como el sexo, la edad, así como la presencia de posibles patologías presentes en el esqueleto. Además de caracteres que a simple vista podemos apreciar: la presencia de fracturas, tipología y localización, así como otras evidencias presentes en el hueso (signos de quemado), e incluso, variables externas que nos permitan realizar la reconstrucción del suceso.

Atendiendo a los diferentes traumatismos que pueden ser observados en los restos óseos procedentes de yacimientos arqueológicos, estos van a constituir una condición patológica, que posibilite la aproximación al modo de vida del individuo, e incluso, según el tipo de laceración, orientarnos hacia una probable causa de muerte.

Sería posible identificar el tipo de lesión desde el punto de vista causa-efecto, de manera que haya sido ocasionado por un acto directo, como resultado de un golpe, o indirecto, por una caída, e incluso por acciones repetitivas a lo largo de la vida del individuo (Judd y Roberts, 1999; Lovell, 2008). Por lo tanto, las diferentes lesiones de origen traumático que pueden ser observadas en un esqueleto se clasifican en antemortem, cuando la lesión ocurre antes de la muerte, apreciándose signos de regeneración ósea, lo que implica supervivencia del individuo, pudiendo existir o no relación con la causa del óbito (Ej. posible muerte del individuo como consecuencia de la infección contraída a consecuencia de la fractura). Perimortem, cuando la fractura analizada se ha producido cerca o en el momento de su muerte, pudiendo ser esta la causa del fallecimiento. En este caso, el material esquelético conserva, en elevada proporción, las mismas propiedades que presentaba en vida. El término postmortem, por el contrario, está relacionado con aquellas fracturas ocasionadas después de la muerte del individuo, por lo que no existe ninguna relación con las causas del fallecimiento. Generalmente, estas fracturas se encuentran asociadas a procesos tafonómicos (Etxeberría, 2003; Isidro y Malgosa, 2003; Dirkmaat et al., 2008; Wieberg et al., 2008; Kranioti, 2015).

Sin embargo, la transición entre antemortem, perimortem y postmortem no es tan sencilla, ya que otros factores como el diámetro de la diáfisis (en el caso de huesos largos), el espesor de la cortical, la relación porcentual entre hueso compacto y esponjoso, la concentración mineral y de colágeno del hueso, la presencia de otro tipo de tejidos y la edad del individuo, entre otros, van a influir en los diferentes tipos de fracturas (Galloway y Zephro, 2005; Wheathley, 2008).

En función del caso que presentamos, centraremos la diferenciación entre perimortem y postmortem. La diferenciación entre un hueso fracturado mucho tiempo después de su muerte y una lesión perimortem es relativamente fácil de distinguir, no ocurre lo mismo en el caso de lesiones producidas justo en el momento de la muerte y las ocasionadas poco después (Moraitis y Spiliopoulou, 2006; Cattaneo y Porta, 2009), de manera que considerando el aspecto morfológico del hueso, la manera de discernir entre ambos tipos de fracturas radica en si la lesión se ha producido en hueso fresco o en hueso seco. Sin embargo, hay que tener en cuenta que el hueso retiene el contenido de humedad, así como su matriz de colágeno durante un tiempo después de la muerte, por lo tanto, la morfología de una fractura asociada a un trauma perimortem, puede persistir dentro del intervalo post mortem (Aufderheide y Rodríguez-Maartçin, 1998; Galloway y Zephro, 2005; Dirkmaat et al., 2008; Wheatley, 2008 Wieberg y Wescott, 2008; Passalaqua y Fenton, 2012).

Diferentes autores (Maples, 1986; Wheatley, 2008; Wieberg y Wescott, 2008) sugieren que las características observadas en una fractura peri mortem pueden ser detectadas en lesiones acaecidas semanas después de la muerte del individuo, no proporcionando un intervalo de tiempo exacto, pudiendo detectarse una serie de cambios en la morfología de la fractura.

Teniendo en cuenta estas características, la primera cuestión que nos planteamos es la existencia de coincidencia del momento de la lesión con el de la muerte. Debido a la ausencia de otro tipo de análisis, nos centraremos en las principales características morfológicas de las lesiones y alteraciones óseas observadas.

Basándonos en los patrones de fractura descritos por Villa y Mahieu (1989); Ortner (2003); Galloway y Zephro (2005); Wheatley (2008); Wieberg y Wescott (2008); Barbian y Sledzik (2008); y Kranioti (2015), son cinco las principales características utilizadas a la hora de discernir entre fracturas perimortem y postmortem:

a) Morfología de la fractura. En hueso fresco la fractura presenta superficie irregular, observándose, por lo general, fracturas concéntricas, circulares y en espiral. Mientras que en el hueso seco las fracturas, generalmente, se presentan perpendiculares $u$ horizontales a la superficie de la lesión.

b) Cambios de coloración de la superficie de fractura, diferenciando entre homogénea, si la lesión se ha producido antes del periodo postmortem o heterogénea, ocurrida tiempo después de la muerte del individuo.

c) Ángulo de fractura, formado en la superficie donde se ha producido la lesión, afectando a la cortical del hueso. Se diferencian ángulos obtusos o agudos, en el caso de fracturas originadas en hueso fresco, y ángulos rectos, cuando la fractura se produce en hueso seco.

d) Superficie de fractura lisa cuando la lesión se ha producido perimortem, y de aspecto serrado en el caso de postmortem. 
e) Contorno de fractura. En este caso, y en lo que respecta a los huesos largos, podemos hablar de fracturas rectas y perpendiculares al eje longitudinal del hueso, asociadas a lesiones postmortem, por el contrario, las lesiones curvadas, o en forma de "V", se asocian a fracturas de tipo perimortem.

Asimismo, en el neurocráneo las lesiones pueden ser catalogadas como fracturas por hundimiento, aplastamiento, incisiones y fracturas lineales (Lovell, 2008), entre otras, presentando las mismas características externas ( $a, b, c$ y d) descritas anteriormente. Simultáneamente, ha de tenerse en consideración el posible desplazamiento de fragmentos óseos que pueden llegar a desprenderse como consecuencia del impacto (Campillo, 2001), así como la presencia o ausencia de fracturas de estallido o líneas de fractura, cuyo origen es la zona de la lesión (Lovell, 2008). Igualmente, han de analizarse signos de supervivencia como pueden ser evidencias de reacción periostítica (respuesta osteoblástica), la formación de pequeñas perforaciones presentes por lo general en la cortical (respuesta osteoclástica), así como el desarrollo de puentes óseos entre dos fragmentos (Barbian y Sledzik, 2008).

\subsection{Evidencias de la acción del fuego en los huesos}

Las alteraciones térmicas observadas en los huesos, pueden darse por causas naturales (accidental) o culturales (intencionadas) (Bennett, 1999), proporcionando evidencias que nos permitan aproximarnos al suceso acaecido.

Factores externos y propios del individuo, como tiempo de exposición, la disposición a la fuente de calor, concentración de oxígeno, los componentes orgánicos e inorgánicos del hueso, la presencia de ropas u otro material que pudiese estar protegiendo al individuo (Imaizumi, 2015; Ellingham et al., 2015), así como la edad de muerte, y la presencia de enfermedades, como la osteoporosis, tienden a influir en los patrones de quemado (Christensen, 2002; Ubelaker, 2009).

El hueso cuando está sometido a una fuente de calor, sufre una serie de transformaciones (Walker et al., 2008; Ellingham et al., 2015): deshidratación, descomposición, inversión y fusión. Es en el proceso de descomposición, cuando se van a producir diferentes alteraciones químicas que implican un cambio de coloración como consecuencia de la exposición a elevadas temperaturas (Thompson, 2004).

Las investigaciones realizadas por diferentes investigadores, entre ellos Etxeberría (1994); Walker et al. (2008); Ellingham et al. (2015) e Imaizumi (2015) han permitido establecer una correlación entre el color del hueso y la temperatura del foco de calor. Basándonos en sus resultados, se ha realizado una única tabla que abarca las distintas temperaturas, las posibles variaciones de color observadas y los resultados en el hueso (Tabla II).

\begin{tabular}{|l|c|c|}
\hline $\begin{array}{c}\text { TEMPERATURA } \\
\left(\mathbf{C}^{\circ}\right)\end{array}$ & $\begin{array}{c}\text { VARIACIÓN } \\
\text { DEL COLOR }\end{array}$ & RESULTADOS \\
\hline $\mathbf{2 0 0}$ & $\begin{array}{c}\text { no se observa } \\
\text { variación del color }\end{array}$ & - \\
\hline $\mathbf{2 0 0 - 3 0 0}$ & amarillento / marrón & - \\
\hline $\mathbf{3 0 0 - 4 0 0}$ & marrón / negro & Hueso Carbonizado \\
\hline $\mathbf{5 0 0 - 6 0 0}$ & gris & - \\
\hline $\mathbf{2 0 0}$ & gris azulado / blanco & $\begin{array}{c}\text { Hueso calcinado } \\
\text { o incinerado }\end{array}$ \\
\hline
\end{tabular}

Tabla 2: Variaciones del color en función de la temperatura de la fuente de calor/ Different colors according to temperature levels.

Los cambios de coloración, pueden proporcionar una rápida aproximación de la temperatura a la que el hueso estuvo sometido. Sin embargo, a medida que aumenta la temperatura, y barajando los diferentes factores externos e internos del individuo mencionados anteriormente, se evidencian otra serie de cambios estructurales, morfológicos, e incluso moleculares que nos indican los diferentes intervalos de temperatura a los que se encontraba expuesto, e incluso nos permite discernir si el proceso de quemado se ha producido en hueso fresco o en hueso seco según el tipo de fragmentación que presentan (Etxeberría, 1994; Ubelaker, 2009; Ellingham, 2015).

Nuestro objetivo consiste en tratar de interpretar el suceso ocurrido, que pudo ocasionar la muerte del individuo, basándonos en la evaluación de las lesiones observadas en cráneo y clavícula, así como en los signos de quemado, intentando establecer una relación directa en el tiempo entre ambas lesiones.

\section{MATERIAL Y MÉTODOLOGÍA}

El material objeto de estudio se trata de un individuo, el cual se encontraba de manera aislada, no hallándose asociado a otros enterramientos (Fig. 3).

El esqueleto no se preservaba completo, faltándole las extremidades inferiores, así como la cintura escapular izquierda, brazo izquierdo y coxal del mismo lado. Por lo tanto, es el lado izquierdo del individuo el que se halla prácticamente incompleto, conservándose, exclusivamente, fragmentos de la $12^{\mathrm{a}} ; 10^{\mathrm{a}} ; 9^{\mathrm{a}}$ y $8^{\mathrm{a}}$ costilla. Sin embargo, el estado de conservación de los restos recuperados era óptimo.

Llama la atención la disposición del individuo en la UE.987 - Fase III, así como las lesiones observadas en cráneo, en la clavícula derecha, y en la octava costilla izquierda. Además, se analizó la cuarte falange media de la mano derecha, ya que presenta indicios de proximidad a una fuente de calor. Sin embargo, no fueron recuperadas las falanges distales.

El análisis de las diferentes fracturas fue realizado macroscópicamente, atendiendo a las diferentes características externas, como son el tipo de fractura, la morfología, coloración, superficie, ángulo y contorno de 


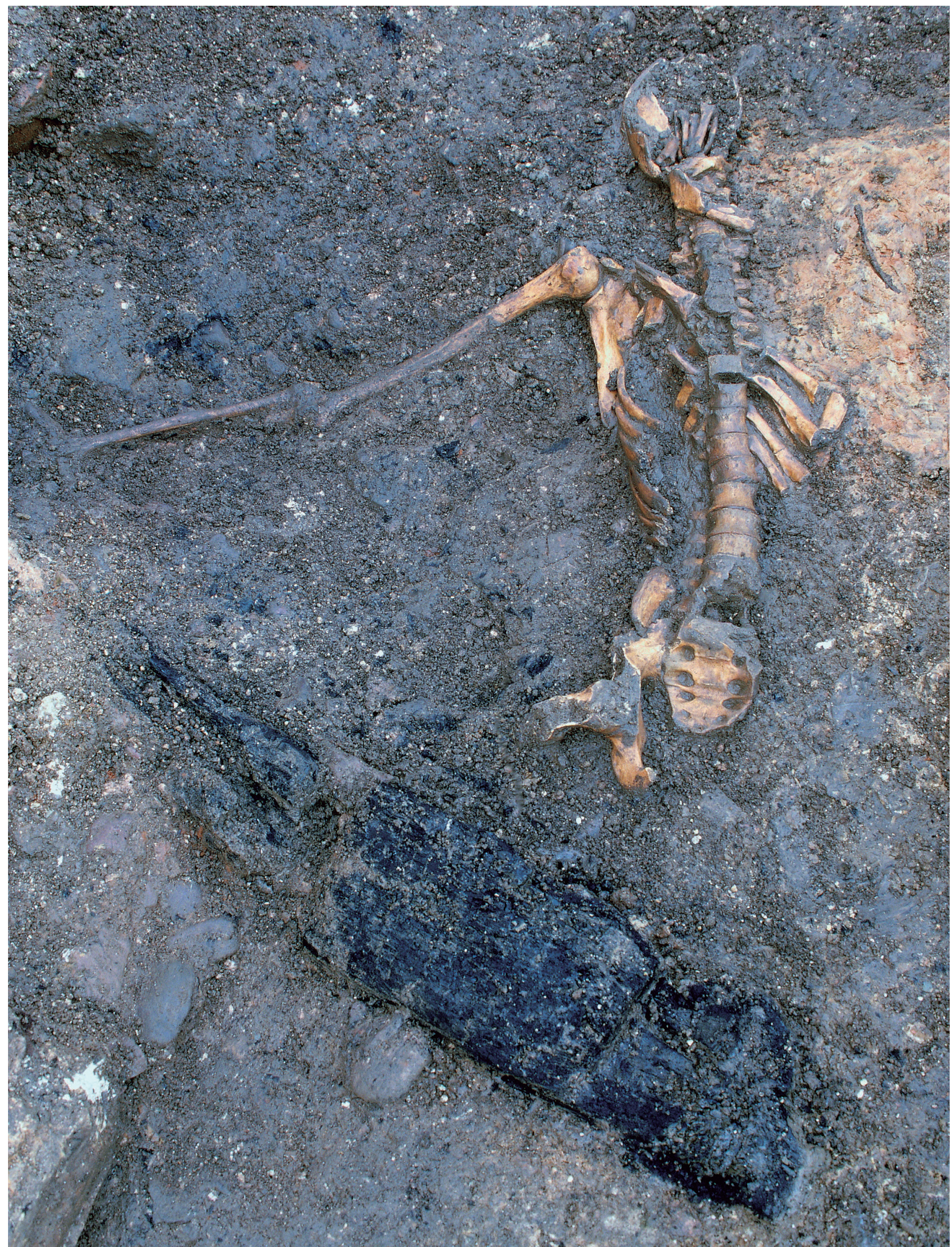

Fig. 3. Localización original del Individuo 6, debajo de los restos carbonizados de una viga. Fotografia: $M^{a}$. Ángeles Sevillano. / Original localitation of the Individual 6 remains, under the carbonise beam. Photography: Mª Ángeles Sevillano. 
la fractura (Villa y Mahieu, 1991; Campillo, 2001; Ortner, 2003; Galloway y Zephro, 2005; Moraitis y Spiliopoulou, 2006; Wheatley, 2008; Wieberg y Wescott, 2008).

Para el análisis de las fracturas del cráneo, además, se tuvieron en cuenta otros parámetros como la presencia o ausencia de fracturas de estallido, fractura conminuta, respuesta osteoblásticas, respuesta osteoclástica, líneas de demarcación, puentes óseos y la tipología de la fractura (Barbian y Sledzik, 2008; Lovell, 2008; Kranioti, 2015)

El estudio la cuarta falange media de la mano derecha, se realizó macroscópicamente, por un lado atendiendo a los cambios de coloración, según los diferentes gradientes de temperatura, y por otro, se tuvo en cuenta la presencia o ausencia de líneas de fractura (Ubelaker, 2009), así como posibles cambios que puedan ser apreciados macroscópicamente en la morfología y textura del hueso.

\section{RESULTADOS}

El individuo analizado es un varón, cuya edad oscila entre los 30 y 35 años, basándonos en los diferentes caracteres cualitativos y cambios morfológicos que han podido observarse en el individuo (Lovejoy et al., 1985; Campillo, 2001; Ubelaker, 2003), y cuya datación, según el material arqueológico adyacente, se sitúa en torno a los siglos IX-X d.C.

En el parietal derecho del cráneo, se aprecia una fractura penetrante e irregular en lo que se puede considerar la zona de impacto, con un tamaño superior en su cara externa respecto a la interna (Fig. 4). Además, se observa desprendimiento de ambas capas de tejido compacto y desplazamiento de un total de 11 fragmentos (fractura conminuta), que por su morfología, nos hace pensar que se hallaban en el interior del cráneo
(Fig. 5). Presenta una coloración homogénea entre la superficie de fractura y la cortical. Dicha superficie muestra un aspecto liso y los ángulos de fractura son agudos. Así mismo, como consecuencia del impacto, se aprecian fracturas de estallido que se prolongan, por un lado, hasta la sutura sagital, y por otro, hacia el hueso temporal del mismo lado (Fig. 4). Cabe destacar que a lo largo y ancho de la lesión craneal analizada no se han observado reacciones periostíticas, respuesta osteoclástica, líneas de demarcación y/o formación de puentes óseos, caracteres indicativos de posible supervivencia del individuo.

La clavícula derecha presenta una lesión de fractura en espiral hacia la mitad de la diáfisis, con morfología en "V", bordes cortantes e irregulares y con aspecto astillado, asimismo, los ángulos de fractura son agudos, observándose además coloración homogénea en toda el área de fractura (Fig. 6). Por otro lado, no se han recuperado los fragmentos que han podido desprenderse en el momento de la lesión y al igual que en e cráneo, no se han observado indicios de supervivencia

Además, se ha observado la formación de callo de fractura, indicativo de supervivencia del individuo, en el tercio proximal de la octava costilla izquierda, no presentando relación con las fracturas analizadas en cráneo y clavícula.

La epífisis distal de la $4^{a}$ falange media de la mano derecha, presenta una coloración negruzca, no observándose fracturas en el hueso, ni alteraciones en la textura (Fig. 7). Sin embargo, no se aprecian a simple vista alteraciones en el resto del individuo como consecuencia de la acción del fuego, o su proximidad a él. Por otro lado, no se han recuperado las falanges distales pudiendo estar mezcladas o incluidas en los restos de material orgánico carbonizado recuperado por los arqueólogos como parte de la UE.923 - Fase IV.
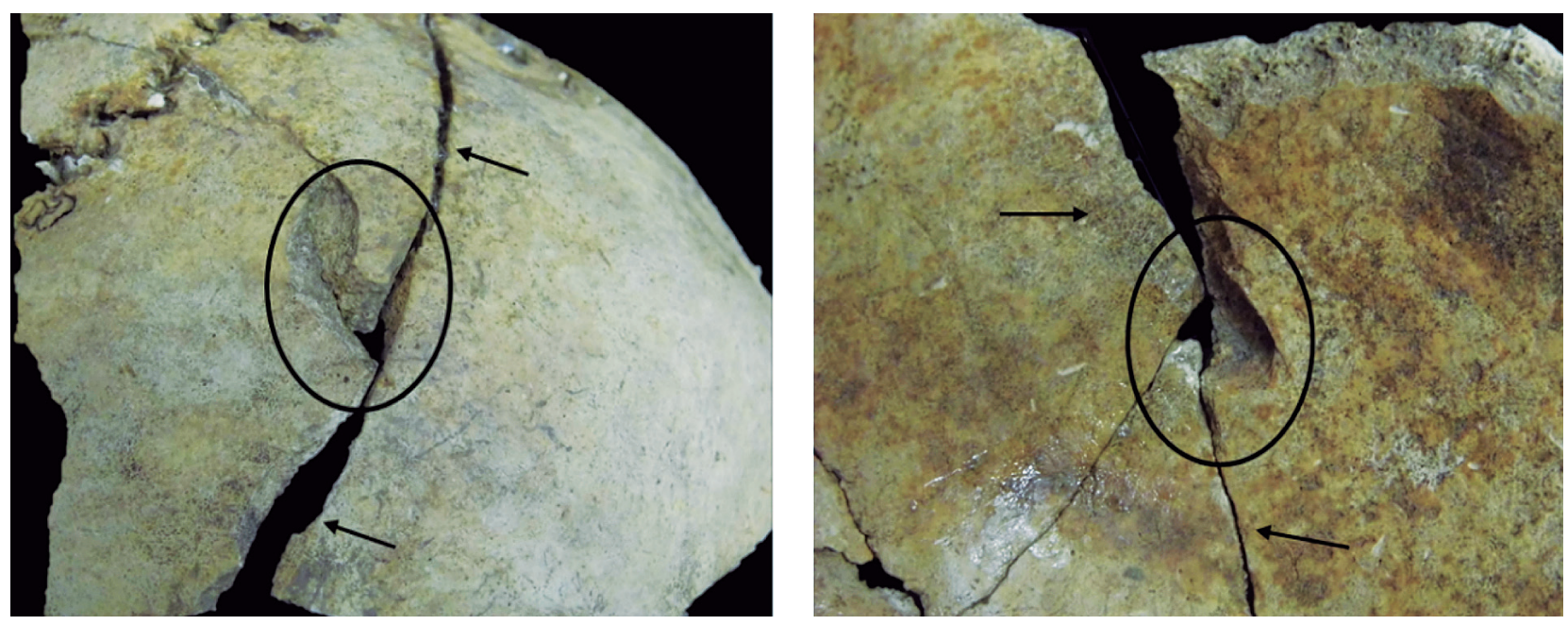

Fig. 4. a) Cara externa de la zona de impacto y líneas de fractura (fracturas de estallido). b) Zona de impacto en la cara interna del cráneo. / a) External side of impact área and fracture lines (linear fracture). b) Impact área from the internal side point of view. 


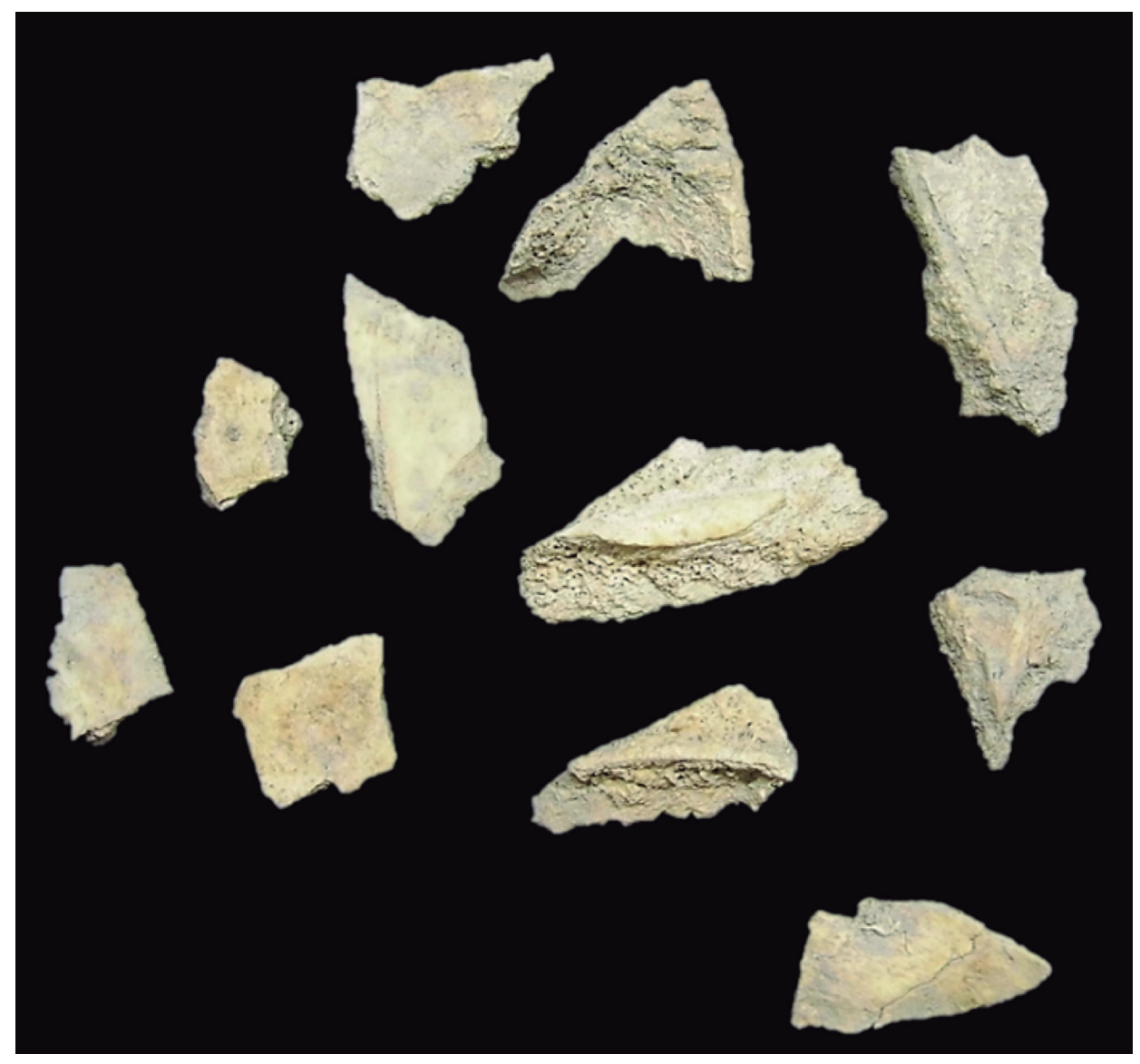

Fig. 5. Fragmentos craneales desprendidos como consecuencia de la lesión. / Cranium fragments detached due to the lesion.

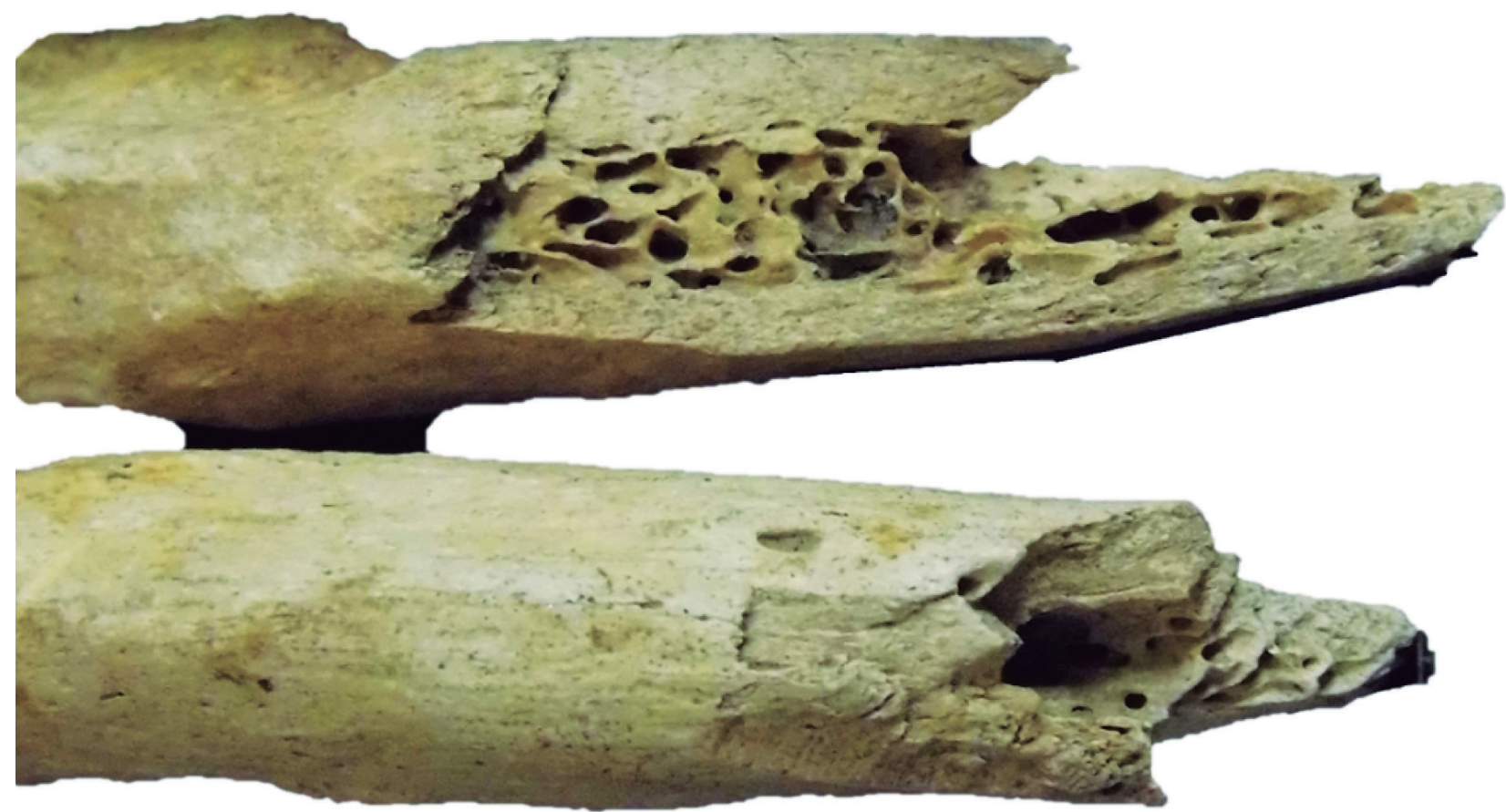

Fig. 6. Fractura de diáfisis clavicular. Se observa la morfología, coloración homogénea, tipo de angulación, superficie, contorno de fractura y descamación. Fracture of the clavicle dyaphasis. 


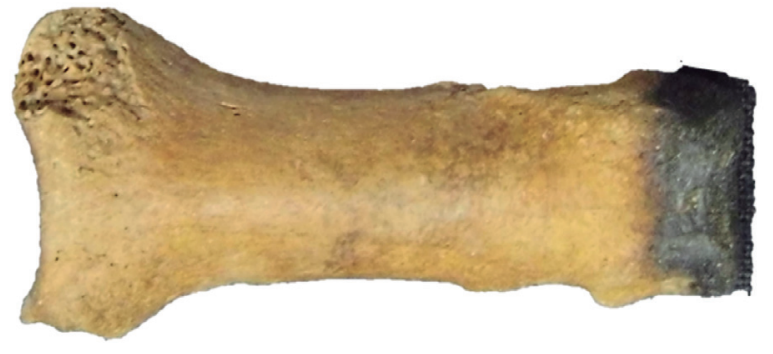

Fig. 7. Falange media con el extremo distal carbonizado. / Meidum phalange with the distal end carbonise.

\section{DISCUSIÓN Y CONCLUSIONES}

Las características diferenciales que se pueden observar en determinadas laceraciones óseas, son fundamentales para conocer cuándo se ha producido dicha o dichas lesiones, y si existe relación directa con la causa de la muerte del individuo. Para ello, es importante diferenciar entre fractura perimortem y postmortem, atendiendo a diferentes factores externos (ej. color, contorno de la fractura, superficie de la fractura, ángulo, etc.), ya que la identificación de la primera nos permite aproximarnos al momento del óbito del individuo (Isidro y Malgosa, 2003).

Aunque la tipología de una fractura radica en si se ha producido en hueso fresco o seco (Ortner, 2003), la respuesta ante una lesión en un hueso largo es distinta a la observada en hueso plano (Campillo, 2001). Concretamente, la reacción del hueso craneal ante un traumatismo, debido a sus características propias, difiere del resto del esqueleto (Campillo, 1992).

Las lesiones en el cráneo por impacto indirecto o caídas desde altura, son relativamente raras. Ambas se encuentran incluidas en un tipo de traumatismo con características comunes, aunque puedan proceder de diferentes escenarios (Kranioti, 2015), lo que hace que este tipo de laceraciones sean difíciles de interpretar (Cohen et al., 2014). Como característica común, estas lesiones muestran una zona de impacto, cuya morfología puede ser redondeada y/o poligonal, con varias líneas de fractura o fracturas de estallido que se forman desde el origen de la lesión (Lovell, 2008; Cohen et al., 2014). Paralelamente, los fragmentos y esquirlas que se desprenden como consecuencia del traumatismo, permanecen dentro de la cavidad craneal (Campillo, 1992; Kranioti, 2015). Según Moritz (1954), las caídas con impacto de la cabeza contra el suelo son más propensas a causar fractura conminuta, implicando un desplazamiento hacia el interior. Por otro lado, Kranioti (2015) se refiere a este tipo de lesiones como fracturas deprimidas, sin especificar si la causa es por caída desde altura o bien por impacto de un objeto. Sin embargo, sí hace referencia a su vinculación con los traumatismos por impacto a baja velocidad, bien sea por un objeto romo o bien por el impacto del propio cuerpo con un objeto romo.
Los patrones de fractura observados en el cráneo llevado a estudio, muestran características macroscópicas relacionadas con fracturas premortem y perimortem (Villa y Mahieu, 1991; Etxeberria et al., 2005; Lovell, 2008; Wheatley, 2008; Wieberg y Wescott, 2008). Además, la presencia de líneas de fractura, así como desprendimiento de fragmentos óseos que en el momento de la lesión habrían quedado en el interior del cráneo, nos orienta hacia un traumatismo con depresión. Por otro lado, la ausencia de puentes óseos, así como reacciones periostíticas nos ha permitido descartar supervivencia.

Sin embargo, como apuntan Dirkmaat et al. (2008), distinguir un traumatismo como consecuencia de un acto violento intencionado, o accidental es difícil en restos humanos antiguos. Por lo que es necesario analizar las características, tanto internas como externas, que rodean al individuo.

Las fracturas de clavícula suelen ocasionarse hacia la mitad de la diáfisis, como resultado de la fuerza de compresión axial directa causada por una caída, o un golpe en el hombro (Wei-Na Ju et al., 2017). Concretamente en nuestro caso, estamos ante una fractura completa en espiral, con pérdida ósea, cuyas características morfológicas y estructurales, así como su coloración implica una fractura perimortem.

Además, los indicios de carbonización observados en la $4^{a}$ falange media es otro dato que nos ha permitido acercarnos más a la hipótesis de muerte accidenta por caída desde altura y como consecuencia de ello se originaron las diferentes fracturas perimortem.

Según Etxebarría (1994), el hueso fresco sometido a una temperatura de hasta $400^{\circ} \mathrm{C}$, durante unos 15 min, alcanza la carbonización. Igualmente, Christensen (2002), Ubelaker (2009), Ellingham et al. (2015) hacen referencia a los cambios de coloración (color negruzco) durante el proceso de carbonización, como consecuencia del quemado de los componentes orgánicos tanto del hueso como del tejido blando.

Asimismo, Ubelaker (2007), en investigaciones realizadas en un grupo de cremaciones, observó cómo varios huesos de los pies se encontraban únicamente chamuscados y con coloración negruzca lo que indica que habían sido menos afectados por el calor, al contrario de lo que ocurría con el resto del individuo, cuyos huesos se presentaban blanquecinos y quebradizos. Indicando, que los huesos sometidos a combustión extrema presentan una serie de patrones de fractura que nos permiten revelar si el hueso se encontraba en estado fresco o seco. Sin embargo, hay que tener en cuenta que el patrón exacto de destrucción del hueso humano ante un foco de calor no está descifrado del todo, ya que son numerosas las variables, propias del individuo y externas, las que van a influir en este proceso. Además, y como dato adyacente al individuo, s el cuerpo se ha quemado en el mismo lugar, han de observarse otros indicios como coloración del terreno, 
ceniza, e incluso, restos de carbón. Estos signos, quedan reflejados con la presencia de material orgánico carbonizado, así como un fragmento de viga en las mismas condiciones de carbonización situados en la zona donde fue hallado el individuo 6.

Por lo tanto, según las características descritas, existe una relación entre las fracturas analizadas, y los signos de quemado observados. Pudiendo considerar ambos hechos simultáneos o cercanos en el tiempo.

\section{INTERPRETACIÓN DEL SUCESO: INDICIOS ARQUEOLÓGICOS Y ANTROPOLÓGICOS}

Atendiendo a los resultados obtenidos durante el estudio antropológico y estratigráfico se plantean dos posibles hipótesis de causalidad que expliquen las causas que llevaron a la muerte de I.6.
Las características morfológicas de las fracturas, nos orientan a interpretar el suceso como causa accidental. Sin embargo, nos hemos planteado dos posibles variantes englobadas dentro de esta causa propuesta. Por un lado, la muerte del individuo pudo producirse como consecuencia de una caída desde altura (Fig. 8), o bien, debido al derrumbe de la cubierta del edificio, así como el material decorativo y el sillarejo de los muros, según se indica en el contexto arqueológico (Fig. 9), quedando en ambos casos el cuerpo tendido sobre su costado derecho en el pavimento. La localización estratigráfica del cuerpo indica, además, que esa caída se produce en un momento en el cual sobre el pavimento del templo todavía no se había depositado material constructivo relacionado con la cubierta.

Teniendo en cuenta la primera hipótesis, la localización de la UE 923 - Fase IV, cubriendo el I.6, nos indica

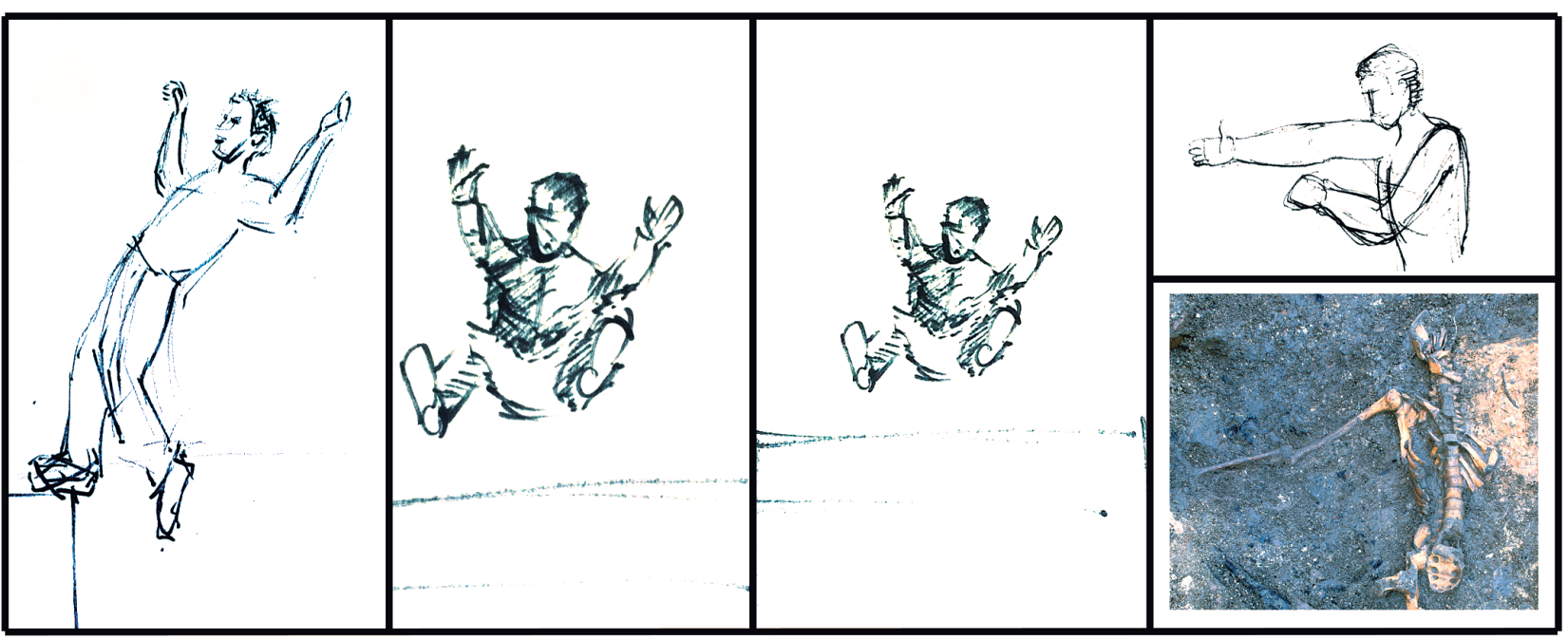

Fig. 8. Reconstrucción teórica de la caída del Individuo 6. Hipótesis 1. Dibujo: Rosa Álvarado Pesquera. / Theorical reconstruction of the Individual 6 fall. Hyphothesis 1. Drawing: Rosa Álvarado Pesquera.

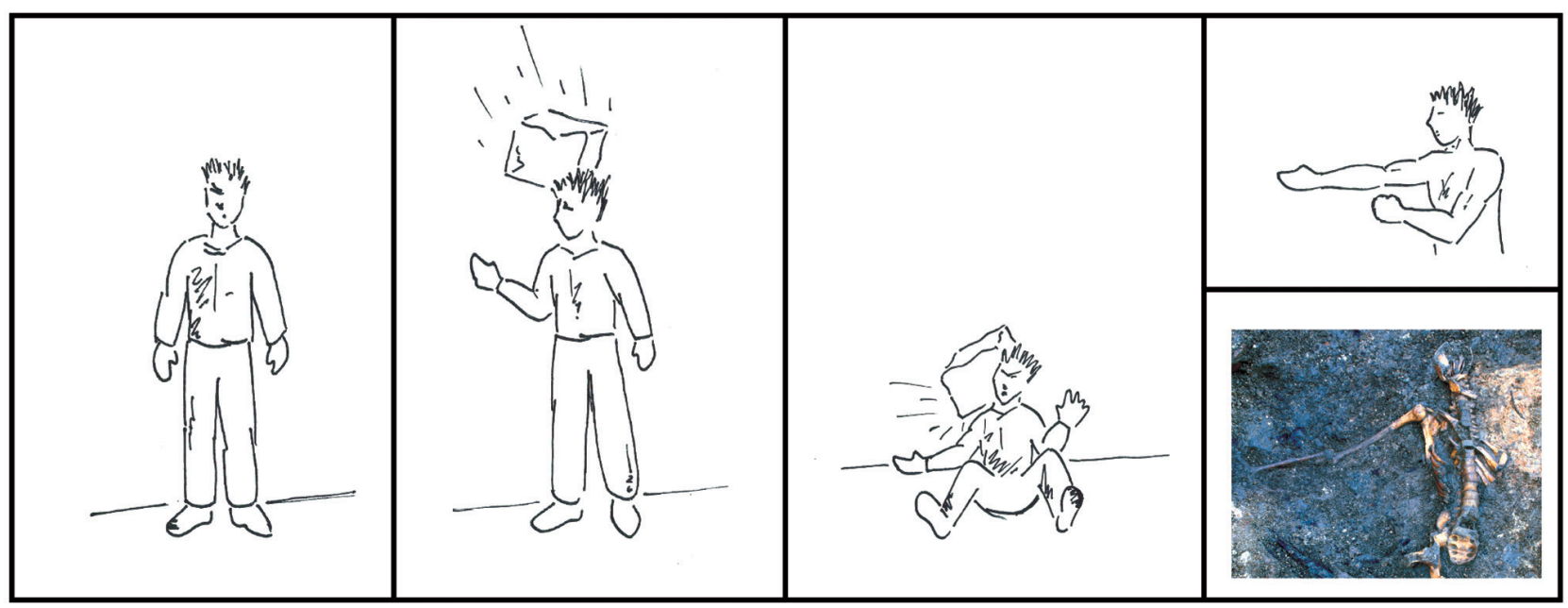

Fig. 9. Reconstrucción teórica de la caída del Individuo 6. Hipótesis 2. Dibujo: Cristina Pérez Gutiérrez. / Theorical reconstruction of the Individual 6 fall. Hyphothesis 2. Drawing: Cristina Pérez Gutiérrez. 
que el colapso de la cubierta ha podido producirse en un momento próximo al accidente. Sin embargo, atendiendo a la segunda hipótesis, sería el material perteneciente a esta UE 923 - Fase IV el que pudo provocar, como consecuencia de ese derrumbe, la muerte del individuo.

La presencia de la viga central de la cubierta carbonizada y su afección sobre el extremo distal de la $4^{a}$ falange media de la mano derecha, constituyen la pieza clave a la hora de proponer una hipótesis acerca de los hechos posteriores al fallecimiento. Atendiendo al análisis de este resto óseo, podemos inferir que el contacto con una fuente de calor se produce, bien inmediatamente después del accidente sufrido por el individuo, o bien en un lapso temporal próximo, durante el cual el hueso se encontraba aún recubierto por tejidos blandos.

La información obtenida en este estudio sobre el fallecimiento de I.6 nos da algunos datos interesantes a la hora de reconstruir el contexto histórico en el que se produce. La situación de la sociedad local, y especialmente de la comunidad religiosa, debía ser especialmente compleja, ya que resulta llamativo que el deceso de una persona y la ruina de un edificio religioso no generase una intervención inmediata en la zona. Cuando el solar se reconvierte en necrópolis de la Catedral románica, se había perdido la memoria, tanto del edificio religioso, como de la muerte de I.6.

\section{AGRADECIMIENTOS}

Los autores quieren hacer patente que esta investigación no se podría haber llevado a cabo sin la colaboración del Museo Romano de Astorga. Este agradecimiento, es especialmente significativo en la persona de $\mathrm{M}^{\mathrm{a}}$. Ángeles Sevillano Prieto, directora del museo y arqueóloga municipal de Astorga, sin cuyo celo científico durante las excavaciones en la plaza Eduardo de Castro, este trabajo hubiera resultado imposible.

También queremos agradecer a Rosa Alvarado Pesquera y Cristina Pérez Gutiérrez por la capacidad de plasmar en papel nuestro relato.

\section{BIBLIOGRAFÍA}

Aufderheide, A.C., Rodríguez-Martín, C., 1998. Traumatic conditions. In: The Cambridge Encyclopedia of Human Paleopathology, 129-50. Cambridge University Press.

Barbian, L. T., Sledzik, P. S., 2008. Healing following cranial trauma. Journal of Forensic Sciences 53(2), 263-268.

Benéitez-González, C., Miguel-Hernández, F., 1993. Relectura arqueológica de la villa romana de Navatejera (León) en Numantia: Arqueología en Castilla y León 6 (1993-1994), 103-126.

Bennett, J.L., 1999. Thermal alteration of buried bone. Journal of Archaeological Science 26(1), 1-8.

Blázquez Martínez, J.M., 1986. La carta 67 de Cipriano y el origen africano del cristianismo hispánico en Homenaje a P. Sáinz Rodríguez, Vol III, 93-102. Madrid.
Campillo, D., 1992. Cicatrización del hueso craneal. Munibe Antropologia-Arkeologia, Supl. 8, 33-49.

Campillo, D., 2001. Introducción a la paleopatología, 295-325. Bellaterra Arqueología.

Carter, H., 1987. El descubrimiento de la tumba Tutankhamon. San Lorenzo Del Escorial.

Christensen, A.M., 2002. Experiments in the combustibility of the human body. Journal of Foresic Science 47(3), 466-470.

Díaz-Martínez, P. C., Martínez-Maza, C., Sanz-Huesma, F. J., 2007. Hispania tardoantigua y visigoda. Madrid.

Dirkmaat, D.C., Cabo, L.L., Ousley, S.D., Symes, S.A., 2008 New Perspectives in Forensic Anthropology. Yearbook of Physical Anthropology 51, 33-52.

Ellingham, S.T.D., Thompson, T.J.U., Islam, M., Taylor, G., 2015. Estimating temperature exposure of burnt bone - A methodological review. Science and Justice 55(3), 181-188.

Etxeberria, F., 1994. Aspectos macroscópicos del hueso sometido al fuego. Revisión de las cremaciones descritas en el País Vasco desde la Arqueología. Munibe Antropologia-Arkeologia 46, 111-116.

Etxeberria, F., 2003. Patología traumática. In: Isidro, A., Malgosa, A. (Eds.), Paleopatología. La enfermedad no escrita, 195208. Masson.

Etxeberria, F., Herrasti, L., Bandres, A., 2005. Muertes violentas determinadas a través de los estudios de paleopatología. Munibe Antropologia-Arkeologia 57, 345-357.

Galloway, A., Zephro, L., 2005. Skeletal trauma analysis of the lower extremity. In: Rich, J., Dean, D.E., Powers, R.H. (Eds.) Forensic Medicine identification and trauma analysis of the thigh, leg and foot, 253-277. Humana Press.

Gil-Tamayo, J.A. (ed.), 2013. Obras completas de San Cipriano de Cartago. Madrid.

Hauschild, T., 1968. La iglesia martirial de Marialba, León en Tierras de León n 9, 21-26. León.

Hauschild, T., 1970. Excavaciones arqueológicas en la basílica paleocristiana de Marialba de la Ribera. León. Informe inédito depositado en el Museo Arqueológico de León.

Cohen, H., Sarie, I., Medlej, B., Bocquentin, F., Toledano, T. Hershkovitz, I., Slon, V., 2014. Trauma to the skull: A historical perspective from the southern levant (4300BCE-1917CE). International Journal of Osteoarchaeology 24(6), 722-736.

Imaizumi, K., 2015. Forensic investigation of burnt human remains. Research and Reports in Forensic Medical Science 5, 67-74.

Ju, W. N., Wang, C.X., Wang, T.J., Qi, B.C., 2017. Fixation of comminute midshaft clavicle fractures with bone fragments separated by soft tissue using a novel double ligature technique. Medicine 96(45), 10-12.

Judd, M.A., Roberts, CH. A., 1999. Fracture Trauma in a Medieval British Farming Village. American Journal of Physical Anthropology 109, 229-243.

Kranioti, E.F., 2015. Forensic investigation of cranial injuries due to blunt force trauma: current best practice. Research and Report in Forensic Medical Science 5, 25-36.

Lovejoy, C.O., Meindl, T.R.P., Mensforth, R., 1985. Chronological metamorphosis of the auricular surface of the ilium: A new method for the determination of adult skeletal age at death. American Journal of Physical Anthropology 68, 1-14. 
Lovell, N.C., 2008. Analysis and interpretation of skeletal trauma. In: Katzenberg, M.A., Saunders, S.R., Biologycal Anthropology of the Human skeleton, 341-386.

Maples, W.R., 1986. Trauma analysis by the forensic anthropologist. In: Reichs, K.J. (Ed.), Forensic osteology: advances in the identification of human remains, 218-228. Charles C Thomas. Springfield, IL.

Moraitis, K., Spiliopoulo, Ch., 2006. Identification and differential diagnosis of perimortem blunt force trauma in tubular long bones. Review. Forensic Science, Medicine, and Pathology 2:4, 221-229.

Moritz, A.R., 1954. The pathology of trauma (2nd ed). Philadelphia: Lea \& Febiger

Muñoz-Villarejo, F., 2001. Excavación arqueológica en la Catedral de Astorga y la iglesia de Santa Marta en La Catedral de Astorga (Actas del Simposio), 327-342. Astorga.

Ortner, D.J., 2003. Identification of pathological conditions in human skeletal remains. Academic Press.

Passalacqua, N.V., Fenton, T.W., 2012. Developments in Skeletal Trauma: Blunt-Force. Trauma. In: Dirkmaat, D.C. (Ed.), A Companion to Forensic Anthropology, 400-411. Blackwell Publishing Ltd.

Pechníková, M., Porta, D., Cattaneo, C., 2011. Distinguishing between perimortem and postmortem fractures: are osteons of any help? Short Communication. International Journal of Legal Medicine 125, 591-595

Sevillano-Fuertes, M.A., Vidal-Encinas, J.M., 2001. Arqueología del entorno de la Catedral de Astorga: la primitiva iglesia de Santa Marta como testimonio de la configuración de un área sacra en La Catedral de Astorga (Actas del Simposio), 25-47. Astorga.

Scheirs, S., Malgosa, A., Sánchez-Molina, D., Ortega-Sánchez, M., Velázquez-Ameijide, J., Arregui-Dalmases, C., Medallo-Muñiz, J., Galtés, I., 2017. New insights in the analysis of blunt forcé trauma in human bones. Preliminary results. International Journal of Legal Medicine 131, 867-875.
Teja, R., 1990. El cristianismo primitivo en la sociedad romana. Madrid.

Thompson, T.J.U., 2004. Recent advances in the study of burned bone and their implications for forensic anthropology. Forensic Science International 146(Supl.), 203-205.

Ubelaker, D. H., 2009. The forensic evaluation of burned skeletal remains: A synthesis. Forensic Science International 183, 1-5.

Ubelaker, D., Rife, J., 2007. The practice of cremation in the Roman-era cemetery at Kenchreai, Greece. Bioarchaeology of the Near East 57, 35-57.

Ubelaker, D.H., 2003. Enterramientos humanos. Excavación, análisis, interpretación. Munibe, Supl. 24. Sociedad de Ciencias Aranzadi.

Villa, P., Mathieu, E., 1991. Breakage Patterns of Human Long bones. Journal of Human Evolution 21(1), 27-48.

Walker, P.L., Miller, K.W.P., Richman, R., 2008. Time, temperature, and oxygen availability: an experimental study of the effects of environmental conditions on the color and organic content of cremated bone. In: Schmidt, C.W., Symes, S.A. (Eds.), The Analysis of Burned Human Remains, 129-135. Academic Press.

Wheatley, P.B., 2008. Perimortem or post-mortem bone fractures? An experimental study of fracture patterns in deer femora. Technical Note. Journal of Forensic Science 53(1), 69-72.

Wieberg, D.A.M, Wescott, D.J., 2008. Estimating the timing of long bone fractures: Correlation between the postmortem interval, bone moisture content, and blunt force trauma fracture characteristics. Journal of Forensic Science 53(5), 1028-1034. 
